\title{
CHARACTERIZATIONS OF FINITE ABELIAN AND MINIMAL NONABELIAN GROUPS
}

\author{
YAKOV BERKOVICH \\ University of Haifa, Israel \\ In memory of Semen L. Gramm (1916-2009)
}

\begin{abstract}
In this note we present the following characterizations of finite abelian and minimal nonabelian groups: (i) A group $G$ is abelian if and only if $G^{\prime}=\Phi(G)^{\prime}$. (ii) A group $G$ is either abelian or minimal nonabelian if and only if $\Phi(G)^{\prime}=H^{\prime}$ for all maximal subgroups $H$ of $G$. We also prove a number of related results.
\end{abstract}

In this note $G$ is a nonidentity finite group and $p, q$ are distinct primes. Our notation is standard for finite group theory (see [3] and [6]).

In what follows we use freely some known properties of $\Phi$-subgroups ([7], see also $[3, \S 1]$ ). For example, if $M \triangleleft G$, then $\Phi(M) \leq \Phi(G)$, and $G$ is nilpotent if and only if $G^{\prime} \leq \Phi(G)$ which is equivalent to normality of all maximal subgroups in $G$ (Wielandt; see Lemma J(d)). It follows from Schur-Zassenhaus' theorem that orders $|G|$ and $|G / \Phi(G)|$ have the same prime divisors (see Lemma J(g)). If $P \in \operatorname{Syl}_{p}(G)$ is $G$-invariant, then $P \cap \Phi(G)=\Phi(P)$ ([1]; see Lemma $\mathrm{J}(\mathrm{a})$ and its proof following Lemma $\mathrm{J}$ (for more general result where $P$ is a normal Hall subgroup of $G$, see [2, Theorem 3.1]). We also use the MillerMoreno-Redei description of minimal nonabelian groups (see Lemma $\mathrm{J}(\mathrm{b}, \mathrm{c})$; for proofs, see [3, Exercise 1.8a] and [6, Lemma 11.2]). For example, if $G$ is a minimal nonabelian group, then $\Phi(G)$ is primary cyclic if $G$ is nonnilpotent, and $G$ is prime-power with $\left|G^{\prime}\right|=p$ if $G$ is nilpotent.

2010 Mathematics Subject Classification. 20D15.

Key words and phrases. Maximal subgroup, abelian, minimal nonabelian, minimal nonnilpotent and Frobenius groups, Frattini subgroup, derived subgroup. 
If $G$ is abelian, then $G^{\prime}=\Phi(G)^{\prime}$ and it appears that this property characterizes abelian groups (Theorem 1 ). If $G$ is either abelian or minimal nonabelian, then $H^{\prime}=\Phi(G)^{\prime}$, and Theorem 3 shows that this property is characteristic for groups all of whose maximal subgroups are abelian.

Let $\Gamma_{1}\left(\Gamma_{1}^{n}\right)$ ) be the set of maximal (nonnormal maximal) subgroups of a group $G$. A group $G$ is not nilpotent if and only it the set $\Gamma_{1}^{n}$ is not empty (Lemma $\mathrm{J}(\mathrm{d})$ ).

In Lemma $\mathrm{J}$ we collected most known results cited in what follows so that our note is self contained modulo Lemma J.

LEMma J. Let $G$ be a finite group.

(a) R. Baer ([1]). If $P \in \operatorname{Syl}(G)$ is $G$-invariant, then $\Phi(P)=P \cap \Phi(G)$.

(b) (Redei; see [3, Exercise 1.8a].) If $G$ is a nilpotent minimal nonabelian group, then $G$ is a p-group, $\left|G^{\prime}\right|=p, \mathrm{Z}(G)=\Phi(G)$ is of index $p^{2}$ in $G$ and one of the following holds:

(i) $p=2$ and $G$ is the ordinary quaternion group,

(ii) $G=\left\langle a, b \mid a^{p^{m}}=b^{p^{n}}=1, a^{b}=a^{1+p^{m-1}}, m>1\right\rangle$ is metacyclic of order $p^{m+n}$

(iii) $G=\left\langle a, b \mid a^{p^{m}}=b^{p^{n}}=c^{p}=1, c=[a, b],[a, c]=[b, c]=1\right\rangle$ is nonmetacyclic of order $p^{m+n+1}$.

If $\exp (G)=p$, then $p>2$ and $|G|=p^{3}$.

(c) (Miller-Moreno; see [3, Lemma 10.8]) If $G$ is a nonnilpotent minimal nonabelian group, then $|G|=p^{a} q^{b}, G=P \cdot Q$, where $P \in \operatorname{Syl}_{p}(G)$ is cyclic, $Q=G^{\prime} \in \operatorname{Syl}_{q}(G)$ is a minimal normal subgroup of $G, \mathrm{Z}(G)=$ $\Phi(G)$ has index $p$ in $P$.

(d) (Wielandt) A group $G$ is nilpotent if and only if $G^{\prime} \leq \Phi(G)$ (or, what is equivalent, $G / \Phi(G)$ is nilpotent).

(e) If $G$ is nilpotent and noncyclic, then $G / G^{\prime}$ is also noncyclic.

(f) ([7]) If a Sylow p-subgroup is normal in $G / \Phi(G)$, then a Sylow $p$ subgroup is normal in $G$. If $H$ is normal in $G$, then $\Phi(H) \leq \Phi(G)$.

(g) $\pi(G / \Phi(G))=\pi(G)$, where $\pi(G)$ is the set of prime divisors of the order of $G$.

(h) ([4]) If $\mathrm{N}_{F}(F \cap H) \neq F \cap H \neq \mathrm{N}_{H}(F \cap H)$ for any two distinct $F, H \in \Gamma_{1}$, then either $G$ is nilpotent or $G / \Phi(G)$ is (nonnilpotent) minimal nonabelian.

(i) If $G$ is a nonabelian $p$-group such that $G^{\prime} \leq \mathrm{Z}(G)$ has exponent $p$, then $\Phi(G) \leq \mathrm{Z}(G)$

(j) (Fitting; see [3, corollary 6.5]) Let $Q$ be a normal abelian Sylow $q$ subgroup of a group $G$. Then $Q \cap \mathrm{Z}(G)$ is a direct factor of $G$.

Let us prove Lemma $\mathrm{J}(\mathrm{a})$. Since $\Phi(P) \leq D=\Phi(G) \cap P($ Lemma $\mathrm{J}(\mathrm{f}))$, it suffices to prove the reverse implication. To this end, one may assume that $\Phi(P)=\{1\}$. Let $H$ be a $p^{\prime}$-Hall subgroup of $G$. Then $P=D \times L$, where $L$ 
is $H$-admissible (Maschke). In that case, $G=(H L) \cdot D$, a semidirect product with kernel $D$, so $D=\{1\}$ since $D \leq \Phi(G)$. Thus, $\Phi(P)=\Phi(G) \cap P$.

Let us prove Lemma J(i). Take $x, y \in G$. Since $G$ is of class 2 , we have $1=[x, y]^{p}=\left[x, y^{p}\right]$ so that $\mho_{1}(G) \leq \mathrm{Z}(G)$, and we obtain $\Phi(G)=G^{\prime} \mho_{1}(G) \leq$ $\mathrm{Z}(G)$.

THEOREM 1. The following conditions for a group $G$ are equivalent:

(a) $G^{\prime}=\Phi(G)^{\prime}$.

(b) $G$ is abelian.

Proof. Obviously, (b) $\Rightarrow$ (a) so it remains to prove the reverse implication. We have $G^{\prime}=\Phi(G)^{\prime} \leq \Phi(G)$ so $G$ is nilpotent (Lemma J(d)); then $G=P_{1} \times \cdots \times P_{k}$, where $P_{1}, \ldots, P_{k}$ are Sylow subgroups of $G$. We have

$$
\begin{gathered}
\Phi(G)=\Phi\left(P_{1}\right) \times \cdots \times \Phi\left(P_{k}\right), \\
G^{\prime}=P_{1}^{\prime} \times \cdots \times P_{k}^{\prime}, \\
\Phi(G)^{\prime}=\Phi\left(P_{1}\right)^{\prime} \times \cdots \times \Phi\left(P_{k}\right)^{\prime} .
\end{gathered}
$$

Equality (1) follows from Lemma $\mathrm{J}(\mathrm{a})$ and (2), (3) are known consequences of (1). Since $\Phi(G)^{\prime}=G^{\prime}$, it follows from (2) and (3) that $\Phi\left(P_{i}\right)^{\prime}=P_{i}^{\prime}$ so $P_{i}$ satisfies the hypothesis for $i=1, \ldots, k$. To complete the proof, it suffices to show that $P_{i}$ is abelian for $i=1, \ldots, k$, so one may assume that $k=1$, i.e., $G$ is a $p$-group. Assume that $G$ is nonabelian of the least possible order. We get $\Phi(G)^{\prime}=G^{\prime}>\{1\}$ so that $\Phi(G)$ is nonabelian. Let $R<\Phi(G)^{\prime}$ be $G$-invariant of index $p$. Since $R<\Phi(G)^{\prime}=G^{\prime}<\Phi(G)$, we get

$$
\Phi(G / R)^{\prime}=(\Phi(G) / R)^{\prime}=\Phi(G)^{\prime} / R=G^{\prime} / R=(G / R)^{\prime},
$$

whence the nonabelian group $G / R$ satisfies the hypothesis so we must have $R=\{1\}$, by induction. In that case, $\left|\Phi(G)^{\prime}\right|=\left|G^{\prime}\right|=p$ so $G^{\prime} \leq \mathrm{Z}(G)$. By Lemma J(i), $\Phi(G) \leq \mathrm{Z}(G)$ so that $\Phi(G)$ is abelian, contrary to what has been said above.

Lemma 2. Let $G$ be a noncyclic p-group and $D=\left\langle\Phi(H) \mid H \in \Gamma_{1}\right\rangle$. Then $D \leq \Phi(G)$ and $|\Phi(G): D| \leq p$ with equality only for $p>2$.

Proof. Since all members of the set $\Gamma_{1}$ are $G$-invariant, we have $D \triangleleft G$ and $D \leq \Phi(G)$ (Lemma $\mathrm{J}(\mathrm{f})$ ). Since all maximal subgroups of the quotient group $G / D$ are elementary abelian, $G / D$ is either elementary abelian or nonabelian of order $p^{3}$ and exponent $p$ (Lemma J(d)). In the first case, $D=\Phi(G)$. In the second case, $p>2$ and $|\Phi(G): D|=|\Phi(G / D)|=p$. In both cases $|\Phi(G): D| \leq p$.

If $H \in \Gamma_{1}$, then $\Phi(G)<H$ so $\Phi(G)^{\prime} \leq H^{\prime}$. Below we consider the extreme case when $\Phi(G)^{\prime}=H^{\prime}$ for all $H \in \Gamma_{1}$.

THEOREM 3. The following assertions for a group $G$ are equivalent: 
(a) $\Phi(G)^{\prime}=H^{\prime}$ for all $H \in \Gamma_{1}$.

(b) $G$ is either abelian or minimal nonabelian.

Proof. Obviously, (b) $\Rightarrow$ (a). Therefore, it remains to prove the reverse implication. In what follows one may assume that $G$ is nonabelian.

(i) The subgroups $\Phi(G), \Phi(G)^{\prime}=H^{\prime}$ are $G$-invariant for all $H \in \Gamma_{1}$. If $\Phi(G)$ is abelian, then all maximal subgroups of $G$ are also abelian, by hypothesis, and $G$ is minimal nonabelian so (b) holds. Next we assume that $\Phi(G)^{\prime}>\{1\}$; then all members of the set $\Gamma_{1}$ are nonabelian. Assume, in addition that $G$ is a $p$-group. Let $R<\Phi(G)^{\prime}$ be $G$-invariant of index $p$ and set $\bar{G}=G / R$; then $\Phi(\bar{G})$ is nonabelian and $\left|\Phi(\bar{G})^{\prime}\right|=p$. Take $H \in \Gamma_{1}$. We have

$$
\Phi(\bar{G})^{\prime}=\Phi(G / R)^{\prime}=(\Phi(G) / R)^{\prime}=\Phi(G)^{\prime} / R=H^{\prime} / R=\bar{H}^{\prime}
$$

is of order $p$ so, by Lemma $\mathrm{J}(\mathrm{i}), \Phi(\bar{H}) \leq \mathrm{Z}(\bar{H})$ and hence $\Phi(\bar{H}) \leq \mathrm{Z}(\Phi(\bar{G}))$ since $\Phi(\bar{H}) \leq \Phi(\bar{G}) \leq \bar{H}$. Setting $\bar{D}=\left\langle\Phi(\bar{H}) \mid H \in \Gamma_{1}\right\rangle$, we conclude that $\bar{D} \leq \mathrm{Z}(\Phi(\bar{G}))$ so $\Phi(\bar{G})$ is abelian since $|\Phi(\bar{G}): \bar{D}| \leq p$, by Lemma 2 , contrary to what has been said already. Thus, if $G$ is a $p$-group, then (a) $\Rightarrow$ (b).

In what follows we assume that $G$ is not a prime-power group. Write $T=\Phi(G)^{\prime}$; then $T=H^{\prime}$ for all $H \in \Gamma_{1}$, by hypothesis. Therefore, all maximal subgroups of $G / T$ are abelian so $G / T$ is either abelian or minimal nonabelian. As in (i), we may assume that $\Phi(G)$ is nonabelian.

(ii) Suppose that $G$ is nilpotent; then $G=P_{1} \times \cdots \times P_{k}$, where $P_{i} \in$ $\operatorname{Syl}_{p_{i}}(G), i=1, \ldots, k, k>1$. It follows from (2) and (3) which are true for any nilpotent group that $\Phi\left(P_{i}\right)^{\prime}=H_{i}^{\prime}$ for all maximal subgroups $H_{i}$ of $P_{i}$ so $P_{i}$ is either abelian or minimal nonabelian, by (i). Then $\Phi\left(P_{i}\right)$ is abelian for all $i$ so that $\Phi(G)$ is abelian, in view of (1), contrary to the assumption. Thus, the theorem is true provided $G$ is nilpotent. Next we assume that $G$ is not nilpotent. In that case, by Lemma $\mathrm{J}(\mathrm{d}), G / T$ is nonnilpotent.

(iii) It remains to consider the case where $G / T$ is minimal nonabelian and nonnilpotent. It follows from the structure of $G / T$ (Lemma J(c)) that $\Phi(G / T)=\Phi(G) / T$ is cyclic. Remembering that $\Phi(G)$ is nilpotent and $T=\Phi(G)^{\prime}$, we conclude that $\Phi(G)$ is cyclic (Lemma J(e)) so abelian, a final contradiction.

COROLlary 4. Suppose that a group $G$ is neither abelian nor minimal nonabelian. Then there exists a nonabelian $H \in \Gamma_{1}$ such that $\Phi(G)^{\prime}<H^{\prime}$.

Proof. We have $\Phi(G)^{\prime} \leq H^{\prime}$ for all $H \in \Gamma_{1}$. Assume that $\Phi(G)^{\prime}=$ $H^{\prime}$ for all nonabelian $H \in \Gamma_{1}$; then $\Phi(G)$ is nonabelian (take a nonabelian $\left.H \in \Gamma_{1}\right)$ so the set $\Gamma_{1}$ has no abelian members. In that case, $\Phi(G)^{\prime}=H^{\prime}$ for all $H \in \Gamma_{1}$, by hypothesis, so that $G$ is minimal nonabelian (Theorem 3 ), contrary to the hypothesis. 
We claim that the following conditions for a group $G$ are equivalent: (a) $H^{\prime} \leq \Phi(G)$ for all $H \in \Gamma_{1}$, (b) either $G$ is nilpotent or $G / \Phi(G)$ is nonnilpotent minimal nonabelian, (c) $\mathrm{N}_{F}(F \cap H) \neq F \cap H \neq \mathrm{N}_{H}(H \cap F)$ for all distinct $F, H \in \Gamma_{1}$. Obviously, (a) $\Leftrightarrow(\mathrm{b})$ and (b) $\Rightarrow(\mathrm{c})$. Next, (b) follows from (c), by Lemma $\mathrm{J}(\mathrm{h})$.

Below we use some known results on Frobenius groups (see [6, §10.2]). Recall that $G$ is said to be a Frobenius group if there is a non-identity $H<G$ such that $H \cap H^{x}=\{1\}$ for all $x \in G-H$. In that case, $G=H \cdot N$ is a semidirect product with kernel $N$, Sylow subgroups of $H$ are either cyclic or generalized quaternion.

LEMMA 5. The following conditions for a nonnilpotent group $G$ are equivalent:

(a) All members of the set $\Gamma_{1}^{n}$ are abelian.

(b) $G / \mathrm{Z}(G)=(U / \mathrm{Z}(G)) \cdot\left(Q_{1} / \mathrm{Z}(G)\right)$ is a Frobenius group with elementary abelian kernel $Q_{1} / \mathrm{Z}(G)=(G / \mathrm{Z}(G))^{\prime}$ and a cyclic complement $U / \mathrm{Z}(G), U \in \Gamma_{1}$.

Proof. If $U, V$ are two distinct abelian maximal subgroups of a nonabelian group $G$, then $U \cap V=\mathrm{Z}(G)$.

Suppose that $G$ satisfies condition (a). Given $H \in \Gamma_{1}^{n}$, set $H_{G}=$ $\bigcap_{x \in G} H^{x}$; then $H_{G}=\mathrm{Z}(G)$, by the previous paragraph. Set $\bar{G}=G / \mathrm{Z}(G)$; then $\bar{G}=\bar{H} \cdot \bar{Q}_{1}$ is a Frobenius group with kernel $\bar{Q}_{1}$ and complement $\bar{H}$. Since $\bar{H}$ is abelian, it is cyclic. There is in $\bar{Q}_{1}$ an $\bar{H}$-invariant Sylow subgroup, by Sylow's theorem. Therefore, since $\bar{H}$ is maximal in $\bar{G}$, the subgroup $\bar{Q}_{1}$ is a $p$-group; moreover, $\bar{Q}_{1}$ is a minimal normal subgroup of $\bar{G}$ so it is elementary abelian. All nonnormal maximal subgroups of $\bar{G}$ are conjugate with $\bar{H}$ (Schur-Zassenhaus) so all members of the set $\Gamma_{1}^{n}$ are conjugate in $G$ (indeed, all members of the set $\Gamma_{1}^{n}$, being abelian, contain $\left.\mathrm{Z}(G)\right)$. Thus, (a) $\Rightarrow$ (b).

Conversely, every group such as in (b), satisfies condition (a). In fact, if $H \in \Gamma_{1}^{n}$, then $\mathrm{Z}(G)<H$. By (b), $H / \mathrm{Z}(G)$ is cyclic so $H$ is abelian.

The proof of Lemma 5 shows that if the set $\Gamma_{1}^{n}$ has at least one abelian member, then the group $G$ has the same structure as in Lemma $5(\mathrm{~b})$.

Corollary 6. A nonnilpotent group $G$ satisfies $\Phi(G)^{\prime}=H^{\prime}$ for all $H \in$ $\Gamma_{1}^{n}$ if and only if $\bar{G}=G / \Phi(G)^{\prime}$ is as in Lemma $5(b)$.

Proof. Suppose that $\bar{G}=G / \Phi(G)^{\prime}$ is as in Lemma $5(\mathrm{~b})$ and $\bar{H}$ is a nonnormal maximal subgroup of $\bar{G}$; then $\bar{H}$ is cyclic, by hypothesis, so $H^{\prime} \leq$ $\Phi(G)^{\prime}$. Since $\Phi(G)<H$, we get $\Phi(G)^{\prime} \leq H^{\prime}$ so $H^{\prime}=\Phi(G)^{\prime}$ and whence $G$ satisfies the hypothesis.

Now suppose that $\Phi(G)^{\prime}=H^{\prime}$ for all $H \in \Gamma_{1}^{n}$. Then all nonnormal maximal subgroups of $G / \Phi(G)^{\prime}$ are abelian so it is as in Lemma 5(b). 
Proposition 7. Suppose that a nonnilpotent group $G$ is not minimal nonabelian and such that $M^{\prime}=\Phi(G)$ for all nonabelian $M \in \Gamma_{1}$. Then

(a) $G=P \cdot Q$ is a semidirect product with kernel $Q=G^{\prime} \in \operatorname{Syl}_{q}(G), P \in$ $\operatorname{Syl}_{p}(G)$ is of order $p, \Phi(G)=\Phi(Q)>\{1\}$. Set $H=P \Phi(G)$. The set $\Gamma_{1}$ consists of two conjugacy classes of subgroups with representatives $H$ and $Q$.

(b) $P^{G}=G$, where $P^{G}$ is the normal closure of $P$ in $G$.

(c) $G / \Phi(Q)$ is minimal nonabelian so that $Q / \Phi(Q)$ is a minimal normal subgroup of $G / \Phi(G)$.

(d) $H^{\prime}<\Phi(Q)$ if and only if $G$ is minimal nonnilpotent.

(e) If $\Phi(Q)$ is abelian, then either $G$ is minimal nonnilpotent or a Frobenius group.

Proof. By hypothesis, all maximal subgroups of $G / \Phi(G)$ are abelian so it is nonnilpotent and minimal nonabelian, by Lemma $\mathrm{J}(\mathrm{d}, \mathrm{c})$, and (c) is proven. It follows that the set $\Gamma_{1}$ is the union of two conjugacy classes of subgroups. We have $\Phi(G / \Phi(G))=\{1\}$ so that $|G / \Phi(G)|=p q^{b}$, where a subgroup of order $p$ is not normal in $G / \Phi(G)$ (Lemma J(b,c)). By hypothesis, the set $\Gamma_{1}$ has a nonabelian member so $\Phi(G)>\{1\}$. By Lemma J $(\mathrm{g}), \pi(G)=$ $\pi(G / \Phi(G))=\{p, q\}$. By Lemma J(f), $Q \in \operatorname{Syl}_{q}(G)$ is normal in $G$. We have $G=P \cdot Q$, where $P \in \operatorname{Syl}_{p}(G)$. By Lemma J(a), $\Phi(Q)=Q \cap \Phi(G)$. By the above, $\Phi(G)=P_{1} \times \Phi(Q)$, where $P_{1}$ is a maximal subgroup of $P$. The subgroup $H=P \Phi(Q) \in \Gamma_{1}$ and all nonnormal maximal subgroups of $G$ are conjugate in $G$ (see the second sentence of this paragraph). Since $P_{1} Q / Q$ is the unique normal maximal subgroup of $G / Q$, it follows that $G / Q \cong P$ is cyclic.

As above, $P_{1} \leq \Phi(G)$ since $|G / \Phi(G)|=p q^{b}$ (here $P_{1}$ is maximal in cyclic subgroup $P$ ). Next, $P_{1} Q=P_{1} \times Q=M \in \Gamma_{1}$. Assume that $P_{1}>\{1\}$ and $M$ is nonabelian. We have $P_{1} \not \leq M^{\prime}=\Phi(G)$, a contradiction. Thus, if $P_{1}>\{1\}$, then $M$ is abelian. Similarly, $P_{1} \not \subset(P \Phi(G))^{\prime}=H^{\prime}$ so that $H$ is also abelian. It follows that $G$ is minimal nonabelian, contrary to the hypothesis. Thus, $P_{1}=\{1\}$ so that $|P|=p$ and $Q \in \Gamma_{1}$. This completes the proof of (a). By Lemma $\mathrm{J}(\mathrm{a}), \Phi(G)=\Phi(Q)$.

Since $Q$, the unique normal maximal subgroup of $G$, has index $p$ in $G$, it follows that $P^{G}=G$, and the proof of (b) is complete.

Suppose that $H^{\prime}<\Phi(G)$. Then $H$ is abelian, by hypothesis. In that case, $\mathrm{C}_{G}(\Phi(G)) \geq H^{G}=G$ so $\Phi(G)=\mathrm{Z}(G)$ since $\mathrm{Z}(G / \Phi(G))=\{1\}$, and we conclude that $G$ is minimal nonnilpotent since $G / \Phi(G)$ is minimal nonabelian. The proof of $(\mathrm{d})$ is complete.

Assume that $\Phi(Q)(=\Phi(G))$ is abelian and $G$ is not minimal nonnilpotent. Then, by $(\mathrm{d}), H=P \Phi(Q)$ is nonabelian so $H^{\prime}=\Phi(Q)$, by hypothesis. In that case, by Lemma $\mathrm{J}(\mathrm{j}), \mathrm{N}_{H}(P)=P$ so that $H$ is a Frobenius group since $|P|=p$. Since $G / \Phi(Q)$ is a Frobenius group, it follows that $G$ is also a 
Frobenius group. This completes the proof of (e) and thereby the proposition.

Let a nonabelian group $G$ be such that $H^{\prime}=\Phi(H)$ for all nonabelian $H \leq G$. Then $G$ has no minimal nonnilpotent subgroups, by Lemma $\mathrm{J}(\mathrm{d})$. It follows that $G$ is nilpotent. Let $A \leq G$ be minimal nonabelian. Then $A$ is a $p$-subgroup for some prime $p$ and $A^{\prime}=\Phi(A)$ has order $p$ and index $p^{2}$ in $A$ (Lemma J(b)) so that $|A|=\left|A^{\prime}\right| p^{2}=p^{3}$. Suppose that $P \in \operatorname{Syl}_{2}(G)$ is nonabelian. Then $P$ is among the 2 -groups described in [8] (see also [5, §90]). Now suppose that $G=Q \times H$, where $Q \in \operatorname{Syl}_{q}(G)$ is nonabelian and $H>\{1\}$. Assume that $Z \leq H$ is cyclic of order $p^{2}$. Then the subgroup $K=Q \times Z$ does not satisfy the hypothesis since $\Phi(Z) \not \leq K^{\prime}$ and $\Phi(Z) \leq \Phi(K)$. Thus, either $G$ is a prime power or $G=Q \times H$, where $Q \in \operatorname{Syl}_{q}(G)$ is nonabelian and $\exp (H)>1$ is square free. It follows that if $H$ is also nonabelian, then $\exp (Q)=q$ and we conclude that $q>2$.

\section{Problems}

1. Study the nonabelian $p$-groups $G$ of exponent $>p$ satisfying $\mho_{1}(\Phi(H))$ $=\mho_{1}(H)$ for all $H \leq G$ (obviously, we must have $p>2$ since for any 2-group $G$ we have $\Phi(G)=\mho_{1}(G)$ ).

2. Classify the $p$-groups $G$ satisfying $H^{\prime}=\Phi(G)^{\prime}$ for all those $H \leq G$ that are neither abelian nor minimal nonabelian.

3. Suppose that $G$ is a $p$-group with $|G / \Phi(G)|=p^{d}$, Given $i \leq d$, let $\Gamma_{i}$ be the set of all normal subgroups $N$ of $G$ such that $G / N$ is elementary abelian of order $p^{i}$. Study the $p$-groups $G$ such that $H^{\prime}=\Phi(G)^{\prime}$ for all $H \in \Gamma_{i}$ (for $i=1$, see Theorem 3).

ACKNOWLEDGEMENTS.

I am indebted to the referee for a number of constructive remarks.

\section{REFERENCES}

[1] R. Baer, Supersoluble immersion, Canad. J. Math. 11 (1959), 353-369.

[2] Y. Berkovich, Alternate proofs of some basic theorems of finite group theory, Glas. Mat. Ser. III 40(60) (2005), 207-233.

[3] Y. Berkovich, Groups of Prime Power Order, Volume 1, Walter de Gruyter, Berlin, 2008.

[4] Y. G. Berkovich and S. L. Gramm, On finite $\Gamma$-quasi-nilpotent groups, Mathematical analysis and its applications, Rostov Gos. Univ., Rostov-Don, 1969, 34-39 (Russian).

[5] Y. Berkovich and Z. Janko, Groups of Prime Power Order, Volume 2, Walter de Gruyter, Berlin, 2008.

[6] Y. Berkovich and E. M. Zhmud, Characters of Finite Groups. Part 1, Translations of Mathematical Monographs 172, AMS, Providence, Rhode Island, 1998.

[7] W. Gaschütz, Über die $\Phi$-Untergruppe endlicher Gruppen, Math. Z. 58 (1953), 160170.

[8] Z. Janko, On finite nonabelian 2-groups all of whose minimal nonabelian subgroups are of exponent 4, J. Algebra 315 (2007), 801-808. 
Y. Berkovich

Department of Mathematics,

University of Haifa,

Mount Carmel, Haifa 31905

Israel

Received: 8.12.2008.

Revised: 5.5.2009. \& 27.6.2009. 